\section{The renal transplant score - a different way of evaluating renal transplant pathology}

and function on

certain criteria such as time of appearance of the kidney after tracer injection, intensity of background, size and homogeneity of tracer uptake by the kidney. Although small, the pilot study could distinguish between hyperacute rejection, acute rejection, chronic rejection and cyclosporin toxicity.

\section{Introduction}

Attempts have previously been made to detect renal transplant pathology scintigraphically, usually with $99 \mathrm{~mm}$ Tc DTPA. The most successful of these is probably the perfusion index. ${ }^{1,2}$ Others include functional imaging $^{3}$ and fractional mean transit time. ${ }^{4}$ With all these aids at hand, diagnosis of renal transplant pathology is still difficult. Serial scintigraphy of both perfusion and function often over a period of days or weeks is necessary for decent monitoring of the progress in renal transplants.

To aid the detection of pathology in renal transplants, a different approach to reading the scintigram was tested, based on renal and background information on the scintigram.

Renal perfusion, uptake and excretion using 99m Tc Glucoheptonate 


\section{The renal transplant score -}

\section{a different way of evaluating}

renal transplant pathology

\section{from page 16}

was scored on selected criteria. The score was compared to the histological diagnosis of various transplants. Glucoheptonate was chosen for interpretation of function (perfusion and early excretion) and parenchymal integrity (delayed views after furosemide injection).

\section{Method}

Eight renal transplants were imaged using 99m Tc Glucoheptonate and biopsied within 24 hours of the scintigraphy for histological diagnosis.

$150 \mathrm{MBq}$ of $99 \mathrm{~m}$ Tc Glucoheptonate were injected into an antecubital vein while a dynamic acquisition of 60 frames of one second was in progress, anterior to the patient. The camera field of view included the renal transplant, large blood vessels, spleen, ureter and bladder.

The first acquisition phase was immediately followed by a second phase of 120 frames of 15 seconds, imaging kidney function. A delayed static view of the transplant was acquired 3 hours later after $20 \mathrm{mg}$ furosemide injection.

The perfusion frames were combined to 15 frames of 4 seconds and the 120 frames of function were combined to 15 frames of 2 minutes. A renogram was generated and frames were displayed, assessed and scored for perfusion, uptake and excretion.

\section{Perfusion criteria (phase 1) included:}

Background score:

0 - Photopenic region in place of kidney

1 - Maximal kidney intensity equal to background

2 - Maximal kidney intensity between background and liver
3 - Maximal kidney intensity same as maximal liver perfusion

4 - Maximal kidney intensity better than maximal liver perfusion

Iliac vessels score:

0 - Photopenic region in place of the kidney

1 - Tracer bolus reaches kidney after it reaches iliac vessels

2 - Tracer bolus reaches kidney before it reaches iliac vessels

Liver score:

0 - Photopenic region in place of the kidney

l - Maximal kidney perfusion after maximal venous liver perfusion

2 - Maximal kidney perfusion before maximal venous liver perfusion

Maximum perfusion score:

$$
4+2+2=8
$$

Function criteria (phase 2) included:

Background score:

0 - Photopenic region in place of kidney

1 - Maximal kidney uptake equal to background

2 - Maximal kidney uptake with prominent background

3 - Maximal kidney uptake with minimal background

Renogram score:

0 - No peak on renogram

1 - Peak after 4 minutes

2 - Peak before 4 minutes

Homogeneity score:

0 - No kidney seen on delayed image

1 - Inhomogenous tracer uptake on delayed image

2 - Homogenous tracer uptake on delayed image

Size score:

0 - No kidney seen

1 - Small kidney
2 - Normally sized kidney

Elimination score:

0 - No kidney seen

1 - Elimination half life from region of interest over the heart > $100 \mathrm{~min}$

2 - Elimination half life from region of interest over the heart of 50-100 $\mathrm{min}$

3 - Elimination half life from region of interest over the heart $<50 \mathrm{~min}$

Maximum function score:

$$
3+2+2+2+3=12
$$

\section{Results}

Table I shows the perfusion scores for the various patients. Table II shows the function scores for the same group of patients. Perfusion and uptake of tracer in acute and hyper-acute transplant rejection are seen in Figures 1

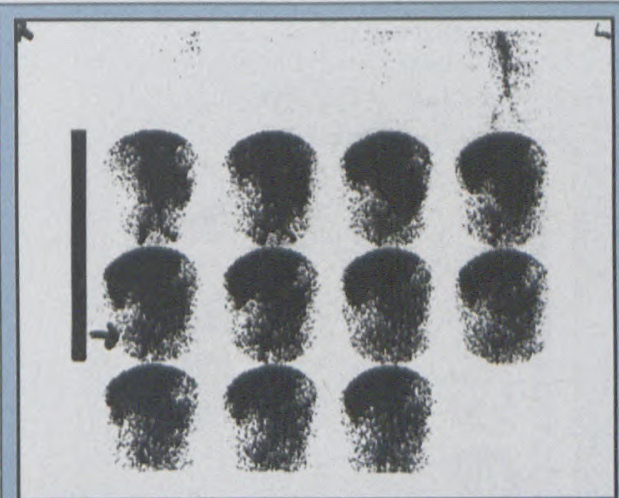

Figure 1a: Fifteen frames of 4 seconds showing the perfusion of a renal transplant undergoing acute rejection.

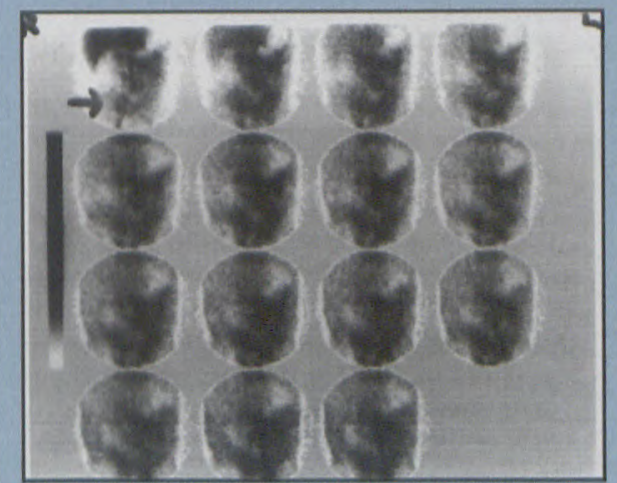

Figure 1b: Fifteen frames of 2 minutes showing the function of a renal transplant undergoing acute rejection 
The renal transplant score -

a different way of evaluating

renal transplant pathology

from page 17

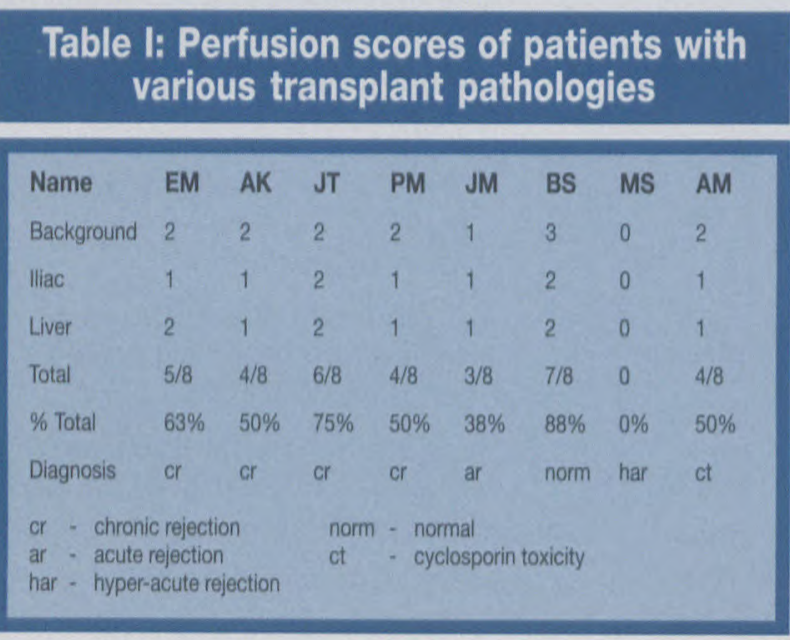

\section{Table II: Function scores of patients with} various transplant pathologies

\begin{tabular}{lllllllll|}
\hline Name & EM & AK & JT & PM & JM & BS & MS & AM \\
Background & 2 & 2 & 2 & 2 & 1 & 2 & 0 & 2 \\
Peak & 1 & 2 & 2 & 2 & 0 & 2 & 0 & 2 \\
Homogeneity & 1 & 1 & 2 & 1 & 1 & 1 & 0 & 2 \\
Size & 2 & 2 & 1 & 1 & 2 & 2 & 0 & 2 \\
Elimination & 1 & 1 & 1 & 1 & 2 & 2 & 0 & 2 \\
Total & $7 / 12$ & $8 / 12$ & $8 / 12$ & $7 / 12$ & $6 / 12$ & $9 / 12$ & $0 / 12$ & $10 / 12$ \\
Percentage & $58 \%$ & $67 \%$ & $67 \%$ & $58 \%$ & $50 \%$ & $75 \%$ & $0 \%$ & $83 \%$ \\
Diagnosis & $c r$ & $c r$ & $c r$ & $c r$ & ar & norm & har & ct \\
cr - chronic rejection & \multicolumn{7}{c}{ norm - normal } \\
ar - acute rejection \\
har - hyper-acute rejection
\end{tabular}

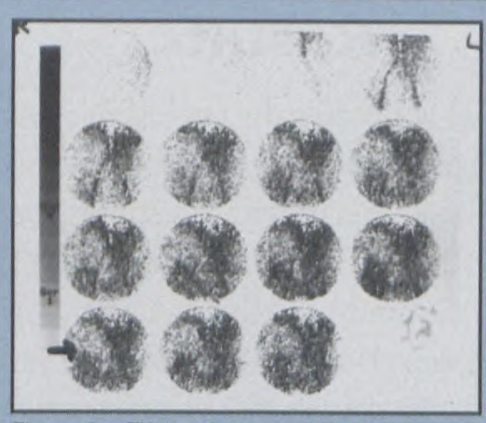

Figure 2a: Fifteen frames of 4 seconds showing the perfusion of a renal transplant undergoing hyper-acute rejection.

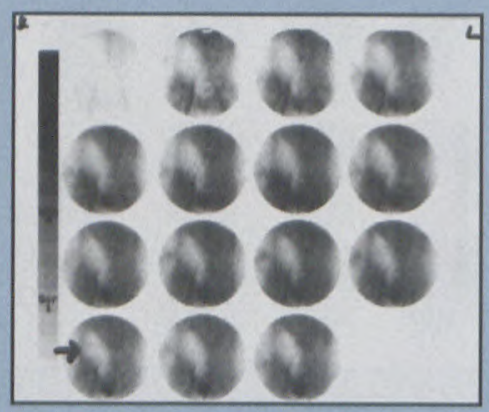

Figure 2b: Fifteen frames of 2 minutes showing the function of a renal transplant undergoing hyper-acute rejection.

and 2. Figure 3 shows the renogram and the half life of tracer in the blood from a region of interest over the heart

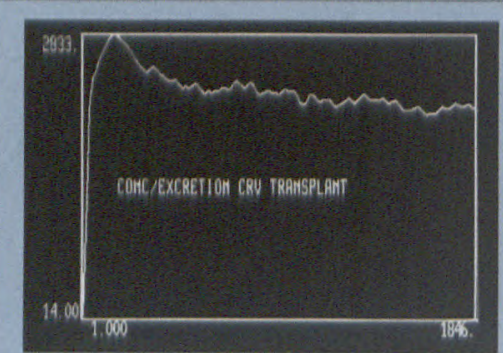

Figure 3a: Renogram of a transplant undergoing acute rejection.

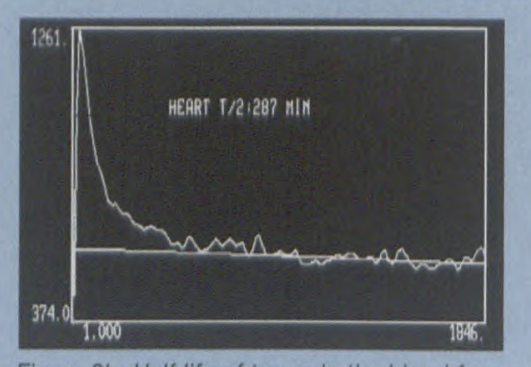

Figure 3b: Half life of tracer in the blood from a region of interest over the heart in a transplant undergoing acute rejection

in a patient with acute rejection. Figure 4 is a bar diagram that compares perfusion and function scores (converted to percent) for various transplant pathologies.

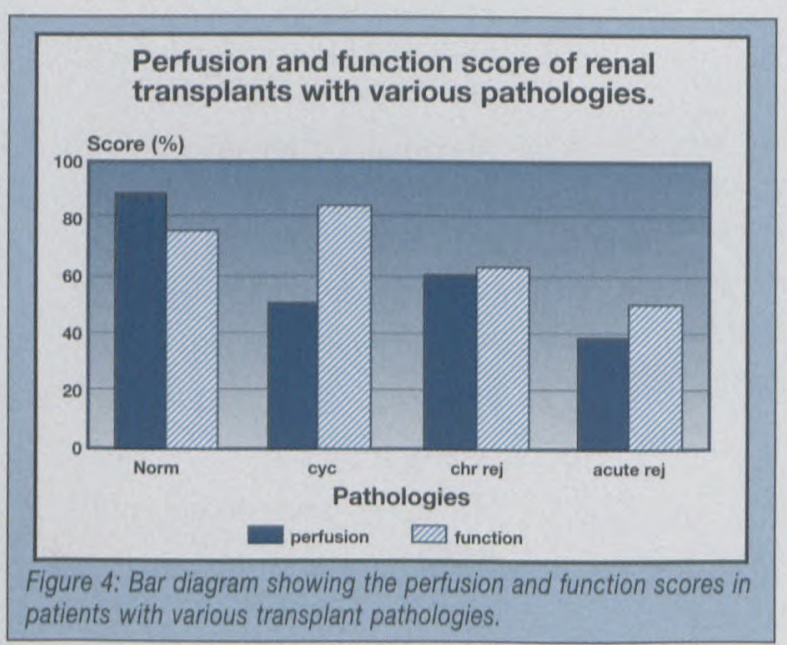

reported ${ }^{5,6,7,8}$ and can be seen in our study. The perfusion score as estimated in our patient group showed a clear difference between the normal transplant, acute and chronic rejection. Attempts have been made previously to use sulphur colloid to predict transplant rejection. These were however unsuccessful. ${ }^{9}$

It has been shown previously that cyclosporin toxicity causes parenchymal tracer retention but does not impair perfusion. ${ }^{10}$ Such patient presented with a moderate perfusion but good function score in our study. In hyper-acute rejection there was no perfusion or uptake of the tracer.

\section{Conclusion}

Our pilot study suggests that a renal transplant score may be a way to distinguish renal transplant pathology. Further evaluation of score criteria and exploration of the method with different tracers is necessary.

\section{Discussion}

Perfusion scintigraphy is a good indicator for acute rejection in renal transplants. This has often been

\section{References}

1. Anaise D, Oster ZH, Atkins HL, Arnold AN, Weis S, Waltzer WC, Rapaport FT. Cortex Perfusion Index: A sensitive detector of acute rejection crisis in transplanted kidneys. J Nucl Med 1986; 27: 1697-1701 